\title{
Anabases
}

ANABASES Traditions et réceptions de l'Antiquité

$30 \mid 2019$

Varia

\section{Freud à Pompéi}

\section{Claude Aziza}

\section{OpenEdition}

Journals

Édition électronique

URL : https://journals.openedition.org/anabases/10324

DOI : 10.4000/anabases.10324

ISSN : 2256-9421

\section{Éditeur}

E.R.A.S.M.E.

\section{Édition imprimée}

Date de publication : 21 octobre 2019

Pagination : 218-221

ISSN : 1774-4296

\section{Référence électronique}

Claude Aziza, «Freud à Pompéi », Anabases [En ligne], 30 | 2019, mis en ligne le 21 octobre 2021,

consulté le 08 novembre 2021. URL : http://journals.openedition.org/anabases/10324 ; DOI : https:// doi.org/10.4000/anabases. 10324

(c) Anabases 

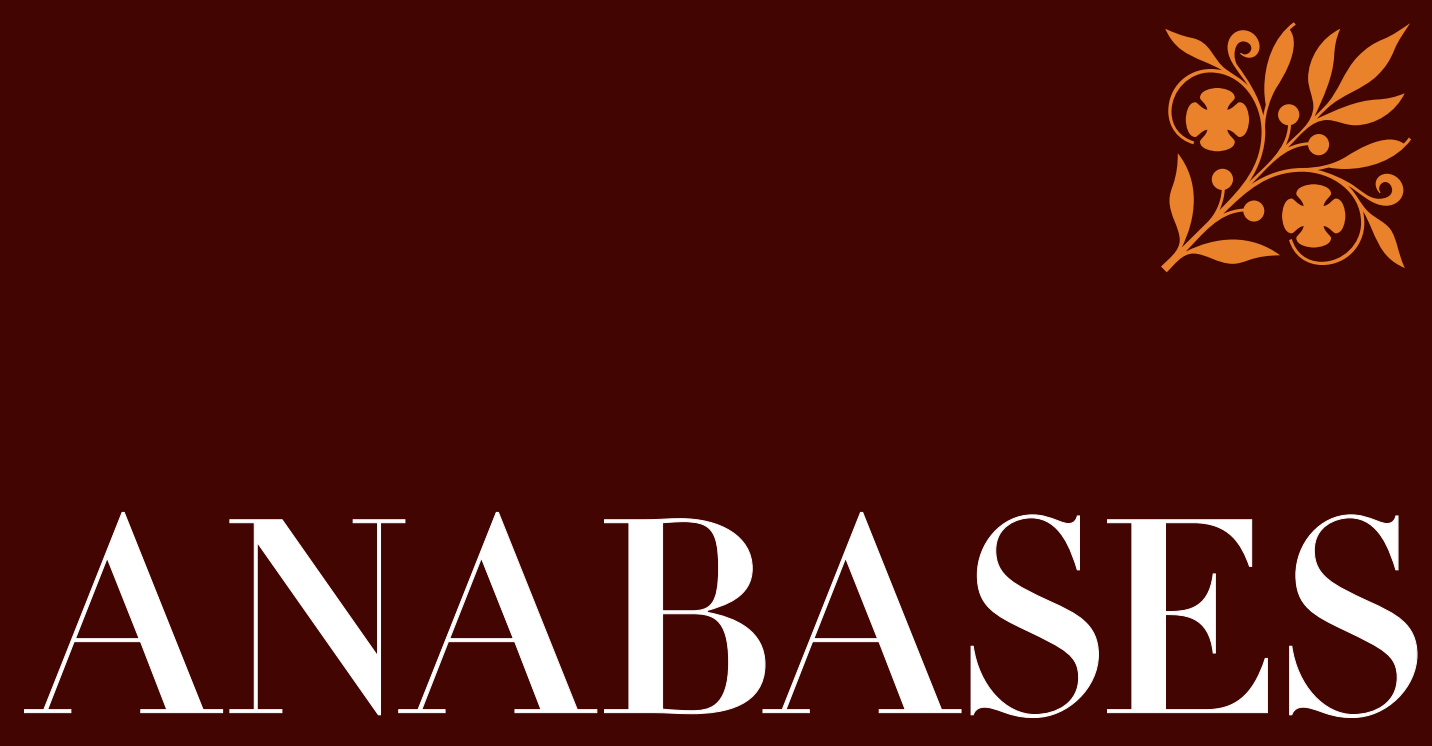

Traditions et Réceptions de l'Antiquité

\section{$\mathrm{N} \times 30$ \\ 2019}

Le sanctuaire de Gournay-sur-Aronde Le pindarisme et l'archéologie musicale Hélène en Égypte Perpétuer Ovide aux $X I V^{e}-X V I I I^{e}$ siècles II classico si fa pop Relire Marcel Detienne Freud à Pompéi 
ANABASES

Traditions et Réceptions de l'Antiquité

Revue de l'équipe de recherche E.R.A.S.M.E.

Université Toulouse-Jean Jaurès (UT2J)

Anabases dispose d'un Comité de lecture international. Chaque article envoyé à la rédaction est soumis, une fois anonymisé, à l'expertise de deux spécialistes qui rendent un rapport écrit. Les deux rapports anonymisés sont transmis à l'auteur qui tient compte des observations en vue de la publication.

\section{Comité SCIENTIFIQue}

Germaine Aujac (université Toulouse-Jean Jaurès : histoire de la géographie et des sciences antiques)

Florence Bouchet (université Toulouse-Jean Jaurès : littérature médiévale)

Hinnerk Brunns (CNRS : histoire économique et sociale ancienne et contemporaine)

Paulo Butti de Lima (université de Bari : historiographie et réception de l'Antiquité)

Luciano CANFora (université de Bari : littérature et histoire anciennes, historiographie)

Giovanna Ceserani (Stanford University : histoire intellectuelle et historiographie de la tradition classique)

Temístocles Cezar (université de Porto Alegre : historiographie moderne)

Serafina Сuомо (University of London, Birkbeck College : histoire des mathématiques et des sciences)

Paul Demont (université de Paris Sorbonne : philologie grecque et héritage classique)

Marie-Laurence Desclos (université de Grenoble II : philosophie de l'Antiquité)

Olivier Devillers (université de Bordeaux 3 - Michel-de-Montaigne : littérature et historiographie latines)

Andrea Giardina (Istituto italiano di scienze umane : histoire du monde romain et de ses réceptions)

Ève Gran-Aymerich (AIBL : histoire de l'archéologie et des transferts culturels)

François Hartog (eHess : historiographie ancienne et moderne)

Geneviève Hoffmann (université de Picardie : histoire des mondes grecs)

Christian JACOB (CNRS/EHESS : histoire comparée et épistémologie des savoirs)

Suzanne Marchand (Louisiana State University : histoire du classicisme et de l'orientalisme)

Wilfried NIPPEL (Humboldt Universität Berlin : histoire et historiographie de l'Antiquité)

Sylvie Pitria (université de Paris I-Panthéon Sorbonne : histoire et historiographie du monde romain)

Stéphane Ratтi (université de Franche-Comté - Besançon : philologie et héritage latin)

Comité de RÉdACtion

Clément Bertau-Courbières, Corinne Bonnet, Laurent bricault, Clément Bur, Adeline Grand-Clément, Anne-Hélène Klinger-Dollé, Véronique Krings, Thibaud Lanfranchi, Pascal Payen, Grégory Reimond, Sarah Rey, Catherine Valenti, Noémie VillacèQue

ÉditeUr RESPONSABLE

Clément Bur

Éditrice ADJOINTE

Catherine VALENTI

Sites Web

http://plh.univ-tlse2.fr

Revues.org : http://anabases.revues.org

Aвonnement et vente aU numéro

Éditions De Boccard - 4, rue de Lanneau - 75005 Paris

info@deboccard.com - www.deboccard.com

Tél. : 0033/(0)143260037 - Fax : 0033/(0)143548583 




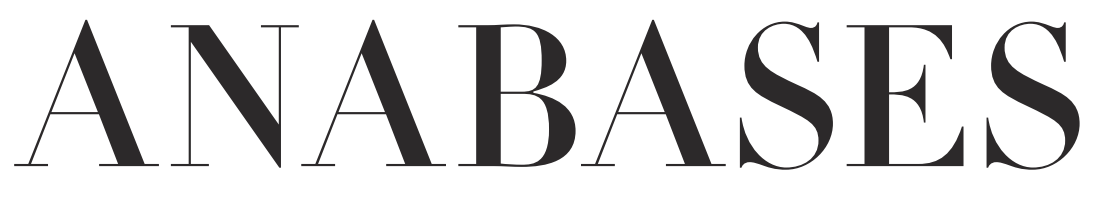

Traditions et Réceptions de l'Antiquité

$$
\begin{aligned}
& N \circ 30 \\
& 2019
\end{aligned}
$$

\section{E.R.A.S.M.E.}

Université Toulouse - Jean Jaurès 



\section{Sommaire}

ํo30 - 2019

\section{Historiographie et identités culturelles}

\section{Carole Quatrelivre}

Le sanctuaire gaulois de Gournay-sur-Aronde (Oise).

Retour sur une découverte exceptionnelle des années I970 . . . . . . . . II

\section{Traditions du patrimoine antique}

Christophe Corbier

Le pindarisme et l'archéologie musicale : style, valeur et authenticité

de la première Pythique à l'époque moderne . . . . . . . . . . . . . . .

Arnaud AmiLIEN

Hélène en Égypte : Hérodote en dialogue avec l’épopée . . . . . . . . . .

\section{Archéologie des savoirs}

Sébastien Cazalas

Au jardin des exempla. Rhétorique et stratégie de l'exemplum antique

dans l'œuvre politique de Jean Juvénal des Ursins (I388-ı473) . . . . . . 7 7

Dossier - Perpétuer Ovide : aspects moraux, éditoriaux,

linguistiques et culturels $\left(\mathrm{XIV}^{\mathrm{e}}-\mathrm{XVIII}^{\mathrm{e}} \mathrm{s}\right.$.)

Francesca Dell'Oro

Introduction $\ldots \ldots \ldots \ldots$. . . . . . . . . . . . . . 89

Hélène Casanova-Robin

L’audace châtiée : Phaéton, Actéon et Icare dans la tradition latine jusqu'à la Renaissance, tours et détours d'un symbolisme . . . . . . . . 9 93 
Dylan Bovet

Le commentaire latin des Métamorphoses d'Ovide : pratiques humanistes et évolutions de Regius-Micyllus (I543) à Burmann-Heinsius (I727) . . . . III

Martine FurNo

Ovide en classe, ou un auteur en éclats . . . . . . . . . . . . . I27

Basil NELIS

D’un Ovide chrétien à un Ovide burlesque, du Moyen Âge au Grand Siècle : continuités et changements dans la traduction et dans l'illustration des Métamorphoses perçus à travers deux éditions du xvII ${ }^{\mathrm{e}}$ siècle . . . . . . . I I43

Olivier ThÉvenAz

Épilogue ....................... I6

\section{Actualités et débats}

Tiphaine-Annabelle BEsnard

2019 : l'année pop des musées d'archéologie. Retour sur l'exposition

romaine Il classico sifa pop. Di scavi, copie e altripasticci . . . . . . . . . . I I7I

\section{Lire, relire la bibliothèque des sciences de l'Antiquité}

Jean-Pierre Albert

Le premier Detienne : une relecture de

“ La notion mythique d"A $\lambda \hat{n} \theta \varepsilon 1 \alpha$ » $\left(R E G\right.$, I96o, p. 27-35) . . . . . . . . . . $\quad{ }_{779}$

Marcel Detienne

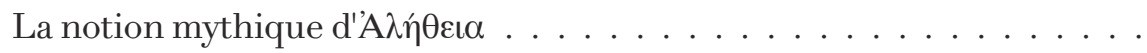

\section{Ateliers de l'histoire}

Antiquités numériques (coordonné par Elodie Guillon) ( $n^{\circ} 1$ )

Élodie GuILlon

Introduction

Jaime Alvar

Le projet EPIDI : Epítetos divinos.

Experiencia religiosa y relaciones de poder en Hispania . . . . . . . . . . . I98 Les mots de l'Antiquité (coordonné par Magali Soulatges) ( $n^{\circ} 10$ )

Jack Thомаs

L’Antiquité dans les toponymes de l'État de New York . . . . . . . . . . . 202

Actualité du théâtre (coordonné par Malika Bastin-Hammou) ( $\left.n^{\circ} 4\right)$

Mathieu FERrand

“ Avons-nous perdu le Soleil ? / Ou l'avons-nous chassé ? » 
Thyeste de Sénèque, traduit par Florence Dupont.

Mise en scène de Thomas Jolly (Avignon, 20I8) . . . . . . . . . . . . . 2 2I4

Voyages et Voyageurs (coordonné par Véronique Krings) ( $n^{\circ} 11$ )

Claude AzIzA

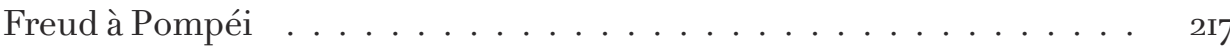

\section{Comptes rendus}

Lucile Arnoux-Farnoux et Polina Kosmadaki (dir.)

Le double voyage : Paris-Athènes (Igrg-Ig39) (Catherine Valenti) . . . . . . 225

Sandra Boehringer et Daniele Lorenzini (dir.)

Foucault, la sexualité, l'Antiquité (Jan Nelis) . . . . . . . . . . . . . . . . 226

Corinne Bonnet, Nicole Belayche, Marlène Albert Llorca,

Alexis Avdeeff, Francesco Massa, Iwo Slobodzianex (dir.)

Puissances divines à l'épreuve du comparatisme. Constructions,

variations et réseaux relationnels (Geneviève Hoffmann) . . . . . . . . . . 228

Shane ButLer (éd.)

Deep Classics, Rethinking Classical Reception (Jan Nelis) . . . . . . . . . . 23o

Zeynep ÇELIK

About Antiquities: Politics of Archaeology in the Ottoman Empire (Jorge Elices Ocón) . . . . . . . . . . . . . . . . . 231

Xavier Deru et Germaine Leman-Delerive (éd),

Franz Cumont, Comment la Belgique fut romanisée (Vivien Barrière) . . . 234

Olivier Devillers, Breno Battistin Sebastiani (éd.)

Sources et modèles des historiens anciens (Arnaud Saura-Ziegelmeyer) . . $\quad 235$

Mara Fazio, Pierre Frantz et Vincenzo De Santis (dir.)

Les Arts du spectacle et la référence antique dans le théâtre

européen (I760-I830) (Arnaud Saura-Ziegelmeyer) . . . . . . . . . . . . . . 237

Jérémy Guedu et Barbara Meazzi (dir.)

La culture fasciste entre latinité et méditerranéité (I880-1940), in Cahiers de la Méditerranée 95, (Andrea Avalli) . . . . . . . . . . . . . . . 239

Marie-Laurence HAAck (éd.), avec la collaboration de Martin MiLler, Les Étrusques au temps du fascisme et du nazisme (Jan Nelis) . . . . . . . . . 24I

Arlene Holmes-Henderson, Steven Hunt et Mai Musié (éd.)

Forward with Classics. Classical Languages in Schools and Communities

(Charlotte Tournier) . . . . . . . . . . . . . . . . . . . . . . .

Daniel Jew, Robin Osborne et Michael Scotт (éd.)

M. I. Finley. An Ancient Historian and his Impact (Hinnerk Bruhns) . . . . $\quad 244$ 
Kostas Kalimtzis,

An inquiry into the philosophical concept of scholê.

Leisure as a Political End (Florent Rouzade) . . . . . . . . . . . . . . . . . 246

Anne-Hélène KLINGER-Dollé

Le De sensu de Charles de Bovelles. Conception philosophique

des sens et figuration de la pensée. Suivi du texte latin du De sensu,

traduit et annoté (Laure Hermand-Schebat) . . . . . . . . . . . . . 248

Egidia Occhipinti

The Hellenica Oxyrhynchia and Historiography:

New Research Perspectives (Anne de Cremoux) . . . . . . . . . . . . . . . . 249

Laurent OLIVIER (dir.)

La mémoire et le temps. L'ouvre transdisciplinaire

d'Henri Hubert (I872-I927) (Sarah Rey) . . . . . . . . . . . . . . . 25I

Gabriella Pinonti et Corinne Bonnet (dir.),

Les dieux d'Homère. Polythéisme et poésie en Grèce ancienne,

Kernos, Supplément 3r. (Sandya Sistac) . . . . . . . . . . . . . . . 253

Rabun TAYLoR, Katherine W. Rinne et S. Kostof

Rome. An Urban History from Antiquity to the Present

(Cyrielle Landrea) . . . . . . . . . . . . . . . . . . . 255

Wyger Velema et Arthur Weststeisn (éd.)

Ancient Models in the Early Modern Republican Imagination

(Paulo Butti de Lima) . . . . . . . . . . . . . . . . . . . 256

Philip WaLsh (éd.)

Brill's Companion to the Reception of Aristophanes

(Malika Bastin-Hammou) . . . . . . . . . . . . . . .

Richard WARren

Art Nouveau and the Classical Tradition (Lucien Calvié) . . . . . . . . . 26o

Jesse Weiner, Benjamin Eldon Stevens et Brett M. Rogers (éd.)

Frankenstein and Its Classics. The Modern Prometheus

from Antiquity to Science Fiction (Mathieu Scapin) . . . . . . . . . . . . . 26r

Nigel G. WiLson

From Byzantium to Italy. Greek Studies in the Italian Renaissance,

deuxième édition (Luigi-Alberto Sanchi) . . . . . . . . . . . . . . 262

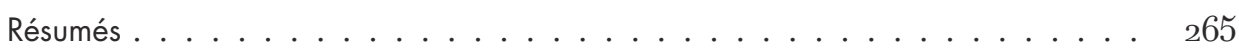

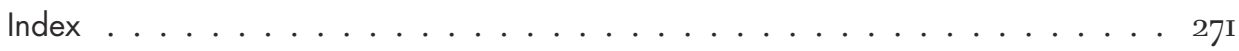




\section{L'atelier de l'histoire : \\ chantiers historiographiques}





\section{Voyages et Voyageurs (12)}

\section{Freud à Pompéi}

\section{Claude AzızA}

“ Je viens d'orner mon bureau de moulages de statues florentines (...). Je rêve à un congrès en terre italienne (Naples, Pompéi). „ C'est ainsi que, le 6 décembre ı896, Freud annonce à son ami Wilhelm Fliess les débuts d'une passion qui le poursuivra toute son existence : celle de l'archéologie. « En fin de compte, écrira-t-il à Stefan Zweig en I93i, j’ai lu plus d'archéologie que de psychologie »!

Depuis i896, Freud a pris l'habitude de partir à l'étranger, la plupart du temps en Italie, de la fin août à la mi-septembre. Mais, pour des raisons complexes qui tiennent à sa relation avec son père et qu'il ne commence à élucider qu'en I897, date de son auto-analyse, il ne peut aborder Rome et ses antiquités. Il doit attendre ıgo pour réaliser " ce rêve longtemps caressé » (lettre à Fliess du Ig septembre Igor). L'année suivante, en Igo2, il s'installe à Sorrente avec son frère Alexandre. Il visite Naples et son musée (le 4 septembre), pousse jusqu'à Capri le 5, puis Paestum, le io. De retour à Naples le ı2, il fait l'ascension du Vésuve le i3. L'étape la plus importante est, on s'en doute, Pompéi, où il reste une journée.

Freud possède des ouvrages sur la cité disparue, parmi lesquels ceux de Johannes Overbeck et d'August Mau. Il en a étudié les rues avec passion : “ J'aspire à quelques belles journées ; d'ailleurs pendant des semaines, dans mes rares heures de liberté, je n'ai rien fait d'autre que couper les pages d'un livre, jouer au solitaire, étudier les rues de Pompéi » (lettre à Fliess du I2 avril I897). Il en a rêvé (lettre au même du 28 avril I897). Il a placé dans son bureau la reproduction d'un "fragment de mur pompéien avec un centaure et un faune », qui le “transporte vers (son) Italie tant désirée » (lettre à Fliess du 24 mai I9oi). À Sorrente, de la terrasse de sa chambre d'hôtel, le Cocumella (pension complète à ı lires par jour!) où Goethe avait également séjourné - Goethe, le poète qu'il aime le plus avec Shakespeare - il peut contempler le magnifique paysage de la baie de Naples. Se mêlent alors, dans son esprit, le parfum des orangers (une lettre écrite à sa famille le 3 septembre Igo2 commence par le vers de l'air de Mignon, l'opéra-comique d'Ambroise Thomas, tiré de la ballade de Goethe : “ Connais-tu le pays où fleurit l'oranger? ») et la beauté des ruines.

Freud marche donc sur les traces de Goethe, le plus illustre de ces pionniers qui partirent voir Pompéi. Il avait été précédé par bien d'autres voyageurs, mais c'est le premier écrivain à voir de près les ruines. Le résultat est parfois bien décevant : quelques notes dans son journal de voyage. Le Ir mars I787, il est à Pompéi qui le «surprend (...) par ses proportions exiguës et rétrécies. Des rues étroites, bien que droites et bordées de trottoirs, de petites maisons sans fenêtres, des chambres éclairées seulement par les 
portes donnant sur les cours et les galeries ouvertes. Même les édifices publics (...) sont plutôt des modèles et des armoires de poupées que des bâtiments ». Le I8 mars, il est à Herculanum, il regrette que « les fouilles n’aient pas été faites bien méthodiquement par des mineurs allemands ». Il est vrai que, trois ans plus tôt, en I784, Winckelmann comptait, dans les quelques cinquante travailleurs attribués aux fouilles - et dont seuls huit étaient au travail lors de sa visite ! -, “ les esclaves d'Alger et de Tunis »...

Goethe sera suivi par bien d'autres, qu'il est impossible ici de tous énumérer. Citons simplement les plus illustres: Chateaubriand en ı804, Germaine de Staël en i8o5, Lamartine en I8II, Stendhal en I8I7, Berlioz en I83ı, Walter Scott, accompagné de William Gell qui venait de publier un mémoire sur la cité (Pompeiana) en i832, Bulwer-Lytton en ı833, Nerval en ı834, Hugo en i835, Dumas en i835, puis en I860, Chassériau en i840 (il exposera son Tepidarium au salon de i853), Dickens en 1846, Gautier en i85o, Flaubert en ı85ı, Taine en 1864, Mark Twain en I869 (il guide un de ces voyages organisés que Thomas Cook a lancés dans les années i85o), Alma-Tadema en i867, Henry James en i873, Nietzsche en I877, Cosima Wagner en I88o (son époux est resté à l'hôtel à Sorrente, il n'a pas voulu voir Pompéi), Miguel de Unamuno en ı889. Jung suivra Freud en igI7 (après la visite, il refusera absolument d'aller à Rome).

$\mathrm{Au}$ moment où Freud visite Pompéi, c'est Ettore Pais qui dirige les fouilles. C'est le premier directeur qui ne soit pas napolitain. Il a succédé, en ı9o, à Giulio de Petra (I875I9oI) qui avait découvert la villa de P. Fannius Sinistor à Boscoreale. Sous la direction de Pais, les premiers sondages stratigraphiques sous le niveau de 79 sont effectués. C'est le coup d'envoi de la recherche d'une Pompéi plus ancienne. Nul doute que Freud ait été impressionné par cette tentative de remonter à plusieurs couches du passé de la cité. À Pompéi, sa joie est telle que, bien plus tard - il a soixante-dix-sept ans - le vieil homme offrira à l'une de ses patientes, la poétesse américaine Hilda Dootlittle - qui s'en souviendra dans Visages de Freud (TR, I977) - une branche d'oranger, en lui montrant sa collection de statuettes antiques.

Deux ans après cette rencontre avec Pompéi, en rgo4, Freud part, toujours en compagnie de son frère Alexandre, pour Athènes. Ils n'y restent que trois jours. Là, sur l'Acropole, se passe un “ incident tout à fait curieux » qu'il évoque, en I927, dans L'Avenir d'une illusion, mais sur lequel il ne s'explique vraiment qu'en Ig36, trois ans avant sa mort, dans “Un trouble de mémoire sur l'Acropole» (une lettre ouverte, restée fameuse, à Romain Rolland, écrite pour l'occasion du soixante-dixième anniversaire de celui-ci) : "Maintenant vous ne vous étonnerez plus que le souvenir de l'incident sur l'Acropole revienne si souvent me hanter depuis que je suis vieux moi-même, que j'ai besoin d'indulgence et que je ne puis plus voyager ». Il a retrouvé là, d'une façon voilée et sublimée par l'art, quelque chose d'un désir incestueux dont, peut-être, en ıgıo, des figures maternelles archaïques, contemplées en Sicile, raviveront le souvenir.

Pour ce voyage, Freud a demandé cette fois à Sandor Ferenczi, son plus récent disciple, de l'accompagner. Les deux voyageurs s'embarquent, en septembre, à Naples pour Palerme, et s'installent à l'Hôtel de France. Ils visitent Sélinonte, où Freud repense à Hannibal, auquel il s'était autrefois pour ainsi dire identifié. Puis ce seront Agrigente et Syracuse. Là, Freud est très impressionné par la statue d'Archimède, qu'il voit près de la source d'Aréthuse; la statue tient de la main droite son fameux miroir parabolique et scrute l'horizon où va apparaître la flotte romaine. L'impression est si forte qu'il fait un rêve bizarre dans la nuit du Io au II octobre (note ajoutée en I9II au chapitre V de L'Interprétation des rêves). Il semble bien qu'il ait considéré Syracuse comme le moment le plus 
important de son voyage, peut-être parce qu'elle était la ville des papyrus, plante dont il avait orné la reproduction de la Gradiva qu'il avait accrochée au mur, dans son cabinet viennois, près du divan. Mais peut-être aussi parce qu'il avait été troublé par la collection de statuettes de femmes que possédait le musée de la ville, ainsi qu'il l'écrivit à Jung.

I9or : Rome. I902 : Pompéi. I904: Athènes. ıgıo : la Sicile. Peu à peu, au cours de ses voyages en Italie et en Grèce, mais surtout à Pompéi, Freud prend conscience de l'étroite parenté qui existe entre l'archéologue et l'analyste. L'un et l'autre essaient d'exhumer ce qui est demeuré enfoui d'une antique civilisation ou d'un désir infantile. “ Tant il est vrai que la réalisation d'un désir infantile est seule capable d'apporter le bonheur ", écrit-il le 28 mai i899, en parlant de la passion qu'éprouvait l'archéologue Heinrich Schliemann, qui découvrit les ruines de Troie, en Asie Mineure, en i87o, puis celles de Mycènes et de Tirynthe. S'il avait été plus amateur de fiction, il aurait pu ajouter : l'auteur de romans historiques. Lui aussi, comme l'analyste, remonte dans le temps pour retrouver dans le passé ce qui, comme le pensaient Gautier ou Mika Waltari, est resté enseveli en luimême, et pour tenter, à travers des destins antiques, de comprendre le sien.

Durant l'été ı9o6, Freud lit une nouvelle de Wilhelm Jensen (I837-I9II) parue en rgo3: Gradiva, fantaisie pompéienne, que Carl Jung lui a conseillée. Immédiatement, il en écrit un commentaire, qui paraîtra en I907 : Le Délire et les rêves dans la Gradiva de Jensen. C'est qu'il a pris conscience qu'، il n'y a pas de meilleure analogie du refoulement - qui tout à la fois rend un élément psychique inaccessible et le conserve - qu'un ensevelissement comme celui qui a été le destin fatal de Pompéi et dont la ville a pu émerger à nouveau par le travail de la pioche ». Cette approche ne rencontra guère d'écho chez Jensen, qui refusa même de rencontrer Freud, mais elle ouvrit une voie féconde à l'application de la méthode analytique aux textes littéraires - et consacra définitivement Pompéi dans son rôle de cité fantasmatique. Freud, quant à lui, gardera toujours une tendresse secrète pour cette femme, la Gradiva. À Rome, en rgo7, il fait enfin sa connaissance : “Pense à ma joie, écrit-il à son épouse Martha, le 24 septembre, en rencontrant aujourd'hui au Vatican, après une si longue solitude, le visage connu d'un être qui m'est cher ; mais la reconnaissance a été unilatérale, car il s'agissait de Gradiva accrochée tout au haut d'un mur. „ Désormais, le moulage du bas-relief montrant une jeune fille traversant une rue figurera dans son bureau - et dans celui de bien des analystes - avec des fresques pompéiennes.

Plus tard, ces belles années fanées dans les brumes du temps, Freud revient plus longuement sur le travail de l'analyste qui

"présente une construction profonde avec celui de l'archéologue, qui déterre une demeure détruite ou ensevelie, ou un monument du passé. Au fond, il lui est identique à cette seule différence que l'analyste opère dans de meilleures conditions et dispose de plus de ressources en matériaux parce que ses efforts portent sur quelque chose qui est encore vivant et non sur un objet détruit (...). Cependant, de même que l'archéologue, d'après des pans de murs restés debout, reconstruit les parois de l'édifice (...), de même l'analyste tire ses conclusions des bribes de souvenirs, des associations et des déclarations de l'analysé. » Ainsi « l'analyste travaille dans de meilleures conditions que l'archéologue (...) [qui] a affaire à des objets détruits (...) [qu'] aucun effort ne réussira à retrouver pour les assembler avec les restes conservés. (...) Il en va tout autrement pour l'objet psychique, dont l'analyste veut recueillir la préhistoire. Ici, il se passe régulièrement ce qui, dans l'objet archéologique, ne s'est produit que dans des circonstances exceptionnelles, comme à Pompéi. » 
Grâce à Freud, la nouvelle de Jensen est devenue universellement célèbre. Son héros est Norbert, un jeune archéologue qui croit retrouver à Pompéi une Pompéienne dont le bas-relief l'a troublé : Gradiva. Il lui parle en latin, elle lui répond dans la même langue. Il comprendra à la fin du récit qu'en fait il a retrouvé une amie d'enfance, bien oubliée depuis des années et dont il était secrètement épris.

Quel dommage que Freud n'ait pas lu Arria Marcella, souvenir de Pompéi, la nouvelle de Gautier (I852)! Nul doute qu'il y aurait trouvé un plus beau sujet d'étude. Car, dans le récit de cette nuit fantastique où le jeune Octavien, en visite avec des amis à Pompéi, se retrouve dans la cité redevenue vivante, il y a matière à une étude psychanalytique en forme. Face à Arria Marcella, qui fascine littéralement sa proie et la laisse sans voix (“ Octavien, pâle, glacé d'horreur, voulut parler mais sa voix resta attachée à son gosier »), le héros de la nouvelle, troublé par le moulage en creux d'un sein aperçu au musée, est persuadé d'en avoir retrouvé la propriétaire, si l'on ose dire. D'un côté, fétichisme du pied, de l'autre, celui du sein... Une lecture psychanalytique, avec toute la part d'arbitraire qu'elle suppose, verrait volontiers dans le récit de Gautier les quatre manifestations du désir et poserait les quatre termes de la phase fantasmatique : désir, interdit, transgression, sanction. Arria Marcella serait la mère interdite et tant désirée et Arrius, qui s’interpose entre elle et Octavien, le père - rival par qui, pourtant, l'enfant accède à l'âge adulte. Pompéi, cité des plaisirs interdits, mère pécheresse punie par le Vésuve, figure paternelle! Au fond, la cure analytique de Norbert est une cure qui a réussi : en bonne analyste, la pseudo-Gradiva est entrée dans le fantasme de son analysant-amoureux, puis, par elle et pour elle, il en est sorti pour revenir à la réalité. L’analyse d'Octavien, en revanche, a échoué : il n'a pas pu échapper à sa névrose et il sera malheureux toute sa vie. N'en fut-il pas ainsi pour Gautier, son créateur?

D'autres récits en lien avec l'Antiquité montrent, avant la lettre, une manifestation troublante de l'inconscient. Le plus célèbre est la nouvelle de Prosper Mérimée (I8o3I87o) : La Vénus d'Ille (I837). On connaît l'histoire de ce malheureux jeune homme qui a mis par inadvertance son anneau nuptial au doigt d'une maléfique statue de Vénus. Celleci, “ tout entière à sa proie attachée ", vient exiger, le jour des noces de son imprudent fiancé - noces avec une autre, bien sûr - l'exécution de sa promesse. Le malheureux y perd la vie. Il ne semble pas que Mérimée ait été à Pompéi et il n’a pu connaître Freud. Pourtant, la rencontre du voyageur qu'il a été au cours de ses inspections archéologiques avec l'Antiquité a quelque chose de troublant. Dans sa nouvelle Le Dernier des Valerii (1875), H. James (I843-ıgi6) qui, lui, revient de Pompéi quand il l'écrit, reprend la même histoire, mais dans un contexte italien. Cependant, elle se termine moins tragiquement que celle de Mérimée.

Retour à Freud. Pour lui, les voyages en Italie et en Grèce se sont, semble-t-il, apparentés à une sorte de quête d'un objet refoulé ou perdu, comme ces amours enfantines sur lesquelles il est resté très discret. Avec Gisela Fluss, fille d'amis de ses parents, qu'il rencontre à $\mathrm{i} 6$ ans, alors qu'elle en a I3. Ou, surtout, avec Pauline, sa nièce, la fille d'Emmanuel son demi-frère, bien plus âgé que lui. On comprend alors l'expression de J.B. Pontalis lorsqu'il écrit, dans sa préface (I986) à l'édition de Le Délire et les rêves dans la Gradiva de Jensen, que Pompéi fut pour Freud le lieu de la « mémoire heureuse ».

Pour quelques autres aussi...

\section{Claude Aziza}

Université de la Sorbonne Nouvelle-Paris III claude.aziza@laposte.net 

ANABASES

Traditions et Réceptions de l'Antiquité

Revue de l'équipe de recherche E.R.A.S.M.E.

Université Toulouse-Jean Jaurès (UT2J)

NoRMES RÉDACTIONNELLES

ANABASES publie des articles dans cinq langues : français, anglais, allemand, italien et espagnol.

Les articles ne dépasseront pas 35000 signes et seront conformes aux normes de la revue,

disponibles sur le site web : http://plh.univ-tlse2.fr

Les articles seront pourvus d'un résumé en français et en anglais, ainsi que de six à huit mots-clés dans ces deux langues.

Les articles pourront être accompagnés de planches en noir et blanc.

Les comptes rendus compteront de 4500 à 6000 signes.

Site web avec Présentation, Sommaire de tous les Numéros et Bulletin d’Abonnement

http://plh.univ-tlse2.fr

Revues.org : http://anabases.revues.org

\section{Courrier}

Pour les articles :

Clément Bur (clement.bur@univ-jfc.fr)

Catherine VALENTI (catherine.valenti@univ-tlse2.fr)

Pour les comptes rendus :

NoémieVILlacÈQue (noemie.villaceque@univ-reims.fr)

Université Toulouse-Jean Jaurès (UT2J)

Équipe P.L.H. - E.R.A.S.M.E. (EA4601)

Maison de la recherche (MdR)

5, allées Antonio Machado

F-31058 Toulouse Cedex 9

Tél. : 0033/(0)5.61.50.25.56 et 57

Fax : 0033/(0)5.61.50.24.90 


\section{Historiographie et identités culturelles}

Carole QuATRELIVRe, Le sanctuaire gaulois de Gournay-sur-Aronde (Oise). Retour sur une découverte exceptionnelle des années 1970

\section{Traditions du patrimoine antique}

Christophe CorbIER, Le pindarisme et l'archéologie musicale : style, valeur et authenticité de la première Pythique à l'époque moderne

Arnaud AmiLien, Hélène en Égypte : Hérodote en dialogue avec l'épopée

\section{Archéologie des savoirs}

Sébastien CAzalas, Au jardin des exempla. Rhétorique et stratégie de l'exemplum antique dans l'œuvre politique de Jean Juvénal des Ursins (1388-1473)

Dossier - Perpétuer Ovide : aspects moraux, éditoriaux, linguistiques et culturels $\left(\mathrm{xIV}^{\mathrm{e}}-\mathrm{XvIII}{ }^{\mathrm{e}} \mathrm{s}\right.$.)

Francesca DelL'Oro, Introduction

Hélène Casanova-Robin, L'audace châtiée : Phaéton, Actéon et Icare dans la tradition latine jusqu'à la Renaissance, tours et détours d'un symbolisme

Dylan Bovet, Le commentaire latin des Métamorphoses d'Ovide : pratiques humanistes et évolutions de Regius-Micyllus (1543) à Burmann-Heinsius (I727)

Martine Furno, Ovide en classe, ou un auteur en éclats

Basil Neurs, D'un Ovide chrétien à un Ovide burlesque, du Moyen Âge au Grand Siècle : continuités et changements dans la traduction et dans l'illustration des Métamorphoses perçus à travers deux éditions du xvir siècle

Olivier ThÉvenaz, Épilogue

\section{Actualités et débats}

Tiphaine-Annabelle BESNARD, 2019 : l'année pop des musées d'archéologie. Retour sur l'exposition romaine Il classico si fa pop. Di scavi, copie e altri pasticci

\section{Relire les classiques des sciences de l'Antiquité}

Jean-Pierre AıBERT, Le premier Detienne : une relecture de « La notion mythique d'A $\lambda \hat{\eta} \theta \varepsilon\llcorner\alpha$ » (REG, 1960, p. 27-35)

Marcel DetIENNE, La notion mythique d'A $\lambda \hat{\eta} \theta \varepsilon \varepsilon \alpha$

\section{L'atelier de l'histoire : chantiers historiographiques}

Antiquités numériques (coordonné par Élodie Guillon) (1)

Élodie GuILLON, Introduction

Jaime ALvar, Le projet EPIDI : Epítetos divinos. Experiencia religiosay relaciones de poder en Hispania

Les mots de l'Antiquité (coordonné par Magali Soulatges) (10)

Jack Tномаs, L’Antiquité dans les toponymes de l’État de New York

Actualité du théâtre (coordonné par Malika Bastin-Hammou) (4)

Mathieu Ferrand, “Avons-nous perdu le Soleil ? / Ou l'avons-nous chassé ? ”

Thyeste de Sénèque, traduit par Florence Dupont. Mise en scène de

Thomas Jolly (Avignon, 2018).

Voyages et Voyageurs (coordonné parVéronique Krings) (11)

Claude Azıza, Freud à Pompéi

\section{Comptes rendus de lecture}

